\title{
The B and Be States of the Star EM Cepheus
}

\author{
Diana Kjurkchieva, ${ }^{1}$ Dragomir Marchev,${ }^{1}$ T. A. A. Sigut,${ }^{2,3}$ and Dinko Dimitrov ${ }^{4}$
}

\begin{abstract}
We present eleven years of high-resolution, spectroscopic observations for the star EM Cep. EM Cep switches between B and Be star states, as revealed by the level of $\mathrm{H} \alpha$ emission, but spends most of its time in the B star state. EM Cep has been considered to be an eclipsing, near contact binary of nearly equal-massed B stars in order to reproduce regular photometric variations; however, this model is problematic due to the lack of any observed Doppler shift in the spectrum. Our observations confirm that there are no apparent Doppler shifts in the wide spectral lines $\mathrm{H} \alpha$ and He I 6678 in either the B or Be star states. The profiles of He I 6678 typically exhibited a filled-in absorption core, but we detected weak emission in this line during the highest Be state. Given the lack of observed Doppler shifts, we model EM Cep as an isolated Be star with a variable circumstellar disk. We can reproduce the observed $\mathrm{H} \alpha$ emission profiles over the eleven year period reasonably well with disk masses on the order of 3 to $10 \times 10^{-11} M_{*}$ in the Be state with the circumstellar disk seen at an inclination of $78^{\circ}$ to the line of sight. From a disk ejection episode in 2014, we estimate a mass loss rate of $\approx 3 \times 10^{-9} \mathrm{M}_{\odot} \mathrm{yr}^{-1}$. The derived disk density parameters are typical of those found for the classical Be stars. We therefore suggest that the EM Cep is a classical Be star and that its photometric variations are the result of $\beta$ Cep or non-radial pulsations.
\end{abstract}

Subject headings: stars: emission-line, Be: binaries: eclipsing; stars: individual (EM Cep)

Accepted for Publication in the Astronomical Journal, 10 June 2016

\section{The Star EM Cepheus}

EM Cep (HD 208392, HIP 108073, SAO 19718, GSC 04266-02575) is a component of the visual binary ADS 15434 (Zasche et al. 2009) and a member of the open cluster NGC 7160, with an age of 18 Myrs and distance of $830 \mathrm{pc}$.

Emission in $\mathrm{H} \alpha$ from EM Cep was first reported by Plaskett \& Pearce (1931) and Merrill et al. (1932). Rachkovskaia (1977) did not find radial-velocity variations of the $\mathrm{H} \alpha$ line in excess of $15 \mathrm{~km} / \mathrm{s}$ or any phasedependent line-profile variations. Hilditch et al. (1982) observed a $\mathrm{H} \beta$ profile with two emission peaks separated by $480 \mathrm{~km} / \mathrm{s}$, placed symmetrically about a deep core. They found that the strengths of $\mathrm{H} \beta$ and $\mathrm{H} \gamma$ sometimes changed by nearly a factor of two within several hours but without a clear sign of duplicity in

\footnotetext{
${ }^{1}$ Department of Physics, Shumen University, 9700 Shumen, Bulgaria

${ }^{2}$ Department of Physics \& Astronomy, The University of Western Ontario, London, Ontario, Canada

${ }^{3}$ Centre for Planetary Science and Space Exploration, The University of Western Ontario, London, Ontario, Canada

${ }^{4}$ Institute of Astronomy and NAO, Bulgarian Academy of Sciences, Tsarigradsko shossee 72, 1784 Sofia, Bulgaria
}

the spectra. Uesugi \& Fukuda (1982) found the atmosphere of EM Cep to be helium-rich. EM Cep is classified as a B1 III-IV shell star with weak emission, and its $v \sin i$ is estimated to be $280-290 \mathrm{~km} / \mathrm{s}$ (Briot 1986, Hilditch et al. 1982).

EM Cep was discovered to be a periodic variable by Lynds (1959), with period of 0.8d and an amplitude of $0.15^{m}$. Thus, EM Cep turned out a rare, short-period Be star (Pustylnik et al. 2005). Due to its almost sinusoidal light curve, EM Cep was suspected to be a closeto-contact binary consisting of two similar early-B stars. Further observations showed the light variability to be quite irregular (Johnston 1970, Rachkovskaia 1975, 1976, Bakos \& Tremko 1975). Flare events in the $R$ band were also observed (Kochiashvili 1999, Kochiashvili et al. 2007, Mars et al. 2010). Molik \& Wolf (2004) found that EM Cep falls on the "blue envelope" of the colour-period diagram of close binaries.

The main peculiarity of EM Cep is that its light variability has not been found to be accompanied by any pronounced radial-velocity or colour variations (Mars et al. 2010). Different explanations have been proposed for this peculiarity: (a) an unstable common envelope around the components of the contact binary 
(Johnston 1970, Bakos \& Tremko 1975, Rachkovskaia 1977, Briot 1986); (b) a magnetic, oblique rotator combined with a non-uniform brightness distribution over the stellar surface (Harmanec 1984, Balona 1990); (c) pulsations of $\beta$ Cep or non-radial type (Rachkovskaia 1976, Hilditch et al. 1982). Kochiashvili et al. (2007) suggested that the star reveals $\lambda$ Eri-type variability. Finally, we note that Pribulla \& Rucinski (2006) found no observational confirmation of a third companion of EM Cep.

The search for a link between spectroscopic and photometric variations is crucial for testing dynamic models of Be stars (Hubert \& Floquet, 1998). Our prolonged observations of EM Cep and their analysis aim to throw additional light on the nature of EM Cep and, perhaps, the Be phenomenon.

\section{The Classical Be Stars}

Classical Be stars are non-supergiant, early-type stars whose spectra have, or have had at some time, one or more Balmer lines in emission (Struve 1931). Decades of study have revealed that the typically doubly-peaked Balmer emission lines originate from a geometrically-thin, equatorial circumstellar disk fed by gas ejected from the surface of rapidly-rotating B star (Porter \& Rivinius 2003). There is also strong observational evidence that these disks are rotationally supported with the disk matter in Keplerian rotation around the central star (Meilland et al. 2007, Oudmaijer et al. 2011, Meilland et al. 2012, Wheelwright et al. 2012). The classical Be stars are particularly interesting due to their fast rotation, with apparent equatorial speeds up to $70-80 \%$ of their critical velocity (Townsend et al. 2004). It is possible they have been spun up by mass transfer in binaries (McSwain \& Gies 2005) or by the internal transport of angular momentum within single stars (Granada et al. 2013).

Besides Balmer emission lines, Be stars are also characterized by excess continuum emission at ultraviolet (UV), optical, and infrared (IR) wavelengths (Gehrz et al. 1974, Dachs et al. 1988, Kaiser 1989, Zorec \& Briot 1991, Dougherty et al. 1994, Carciofi \& Bjorkman 2006).

Presently, the family of classical Be stars numbers over 2000 known members as catalogued in the Be Star Spectra database (BeSS1, Neiner et al. 2011). Raddi et al. (2015) present a catalogue of a further 247 photometrically and spectroscopically confirmed fainter classical Be stars $\left(13^{m} \leq r \leq 16^{m}\right)$ in the direction of the Perseus Arm of the Milky Way.

The evolutionary status of classical Be stars is debatable (Mermilliod 1982, Slettebak 1985). Martayan et al. (2007) found that the evolution of the rotation speed with age is mass and metallicity dependent and concluded that the Be phenomenon appears earlier in the main sequence phase at higher stellar masses $(\sim 12$ $\mathrm{M} \odot$ ) and earlier spectral types, while it is delayed in later B-types. On the other hand, observations of Galactic-field, bright classical Be stars show a flat distribution across the B sub-types (Zorec \& Briot 1997).

The observational signatures of the Be stars, continuum excess and line emission, are nowadays reproduced successfully by radiation from a viscous decretion disk consisting of dense $\left(\mathrm{n}_{e} \sim 10^{12} \mathrm{~cm}^{-3}\right)$ and hot $\left(\mathrm{T}_{e} \sim 10000 \mathrm{~K}\right)$ gas (Lee et al. 1991, Porter 1999, Okazaki 2001, Bjorkman \& Carciofi 2005, Carciofi 2011, Carciofi et al. 2012).

\section{Spectral Observations of EM Cep}

New spectral observations were carried out by the $2 \mathrm{~m}$ RCC telescope. We used a CCD Photometrics AT200 camera with an SITe SI003AB 1024x1024 pixel chip mounted on the Coudé spectrograph (grating B\&L632 $/ 14.7^{\circ}$ ) on the $2 \mathrm{~m}$ telescope of the National Astronomical Observatory at Rozhen, Bulgaria. The exposure time was $15 \mathrm{~min}$. All stellar integrations were alternated with Th-Ar comparison source exposures for wavelength calibration. The resolution of the spectra is 16400 , and most spectra have a $\mathrm{S} / \mathrm{N}$ ratio in the range 150-250. Initially, EM Cep was observed in the spectral range centered on $\mathrm{H} \alpha$, but after July 2005 , the spectral range was changed to include the He I 6678 line, $2 \mathrm{p}^{1} \mathrm{P}^{\mathrm{o}}-3 \mathrm{~d}^{1} \mathrm{D}$.

The reduction of the spectra was performed using IRAF (Tody 1993) packages for bias subtraction, flat fielding, cosmic ray removal, and one-dimensional spectrum extraction. The spectra were continuum normalized in the region around $6500 \AA$.

Table 1 shows dates of our observations, the number of spectra, and the observing phase calculated according to the ephemeris

$$
J D(\operatorname{Min} \mathrm{I})=2452500.7420+0.806178 E,
$$

from Kreiner et al. (2001).

\section{Qualitative analysis of the spectra}

Our 11 years of observations reveal that the spectra of EM Cep did not change significantly within a single night but differed significantly over longer times. Figures A1 and A2 in the Appendix chronologically show representative spectra from each observational night, while Figures 1 and 2 show sample spectral corresponding to the $\mathrm{Be}$ and $\mathrm{B}$ states.

To quantify the behaviour of $\mathrm{H} \alpha$ over the 11 year period, we measured the following line parameters from 
Table 1: Journal of observations

\begin{tabular}{lcl}
\hline \hline Date & Number of Spectra & Phase \\
\hline 2004 Aug 24 & 18 & $0.81-0.114$ \\
2004 Aug 25 & 6 & $0.04-0.43$ \\
2005 Apr 15 & 11 & $0.34-0.47$ \\
2005 July 28 & 25 & $0.11-0.47$ \\
2005 July 29 & 1 & 0.42 \\
2005 Aug 29 & 7 & $0.76-0.87$ \\
2005 Aug 30 & 4 & $0.96-0.01$ \\
2006 Feb 07 & 8 & $0.74-0.83$ \\
2006 June 21 & 2 & $0.08-0.09$ \\
2006 June 22 & 2 & $0.27-0.29$ \\
2006 July 18 & 10 & $0.53-0.71$ \\
2006 July 19 & 3 & $0.76-0.79$ \\
2007 Aug 8 & 5 & $0.40-0.48$ \\
2007 Dec 18 & 3 & $0.05-0.18$ \\
2007 Dec 19 & 2 & $0.05-0.17$ \\
2010 May 04 & 6 & $0.7-0.77$ \\
2010 May 07 & 4 & $0.71-0.74$ \\
2010 Sept 18 & 1 & 0.78 \\
2011 Apr 11 & 1 & 0.291 \\
2011 Sept 19 & 1 & 0.60 \\
2011 Dec 04 & 1 & 0.89 \\
2012 Jan 03 & 2 & $0.96-0.99$ \\
2012 Jan 04 & 1 & 0.26 \\
2012 Jan 05 & 1 & 0.42 \\
2012 June 30 & 1 & 0.44 \\
2012 Aug 07 & 1 & 0.41 \\
2012 Sept 08 & 2 & 0.195 \\
2013 Apr 21 & 4 & $0.35-0.39$ \\
2013 Aug 25 & 3 & $0.47-0.67$ \\
2013 Oct 24 & 1 & 0.83 \\
2013 Dec 18 & 2 & $0.98-0.99$ \\
2014 June 06 & 3 & $0.096-0.121$ \\
2014 July 8 & 2 & 0.64 \\
2014 July 9 & 1 & 0.81 \\
2014 Aug 08 & 2 & 0.12 \\
2014 Sept 30 & 2 & $0.47-0.67$ \\
2014 Nov 11 & 2 & $0.47-0.67$ \\
2014 Dec 13 & 1 & 0.83 \\
2015 Jan 01 & 3 & $0.98-0.99$ \\
\hline
\end{tabular}

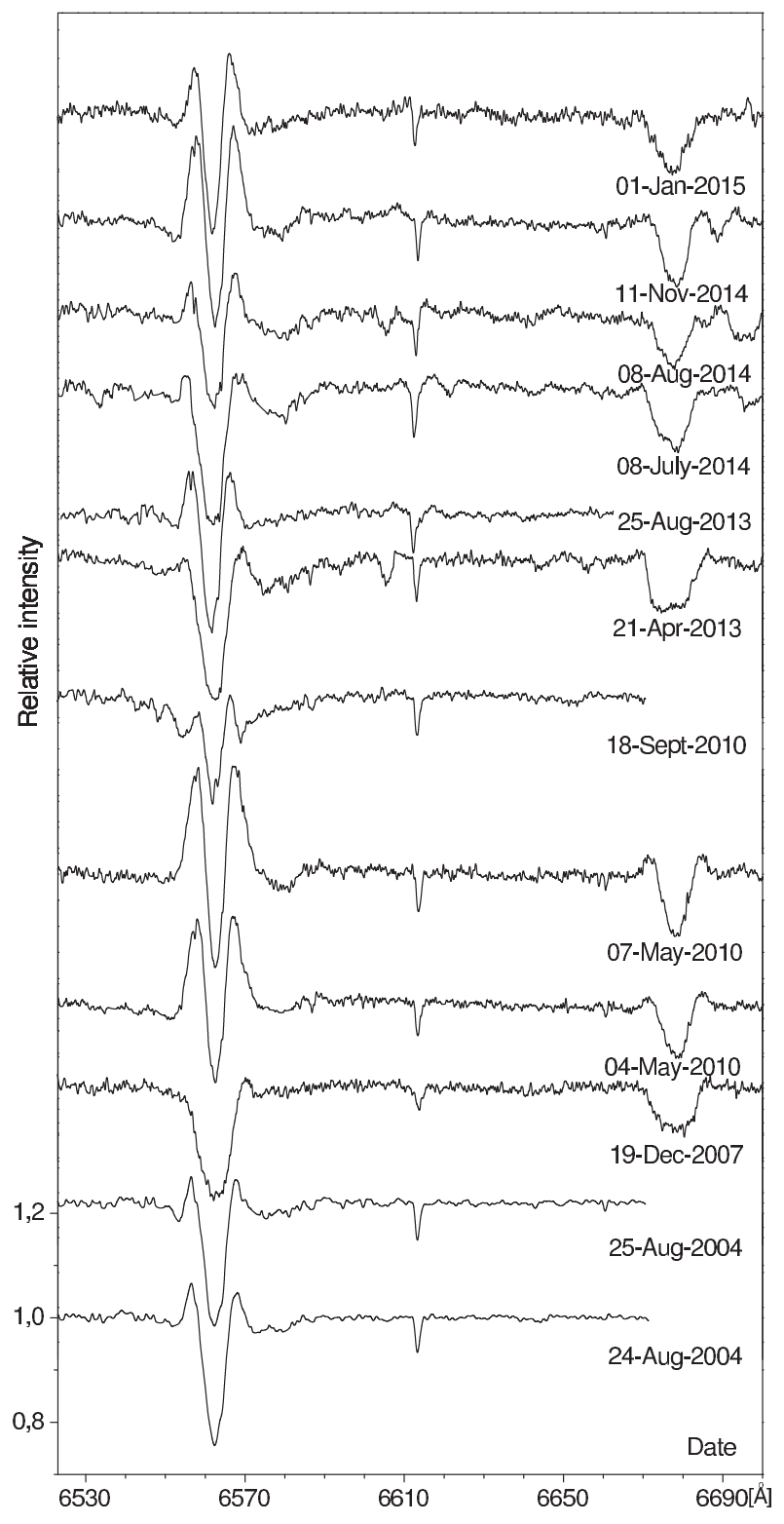

Fig. 1.- A Sample of EM Cep spectra in the Be state. The spectra have been offset for clarity. 


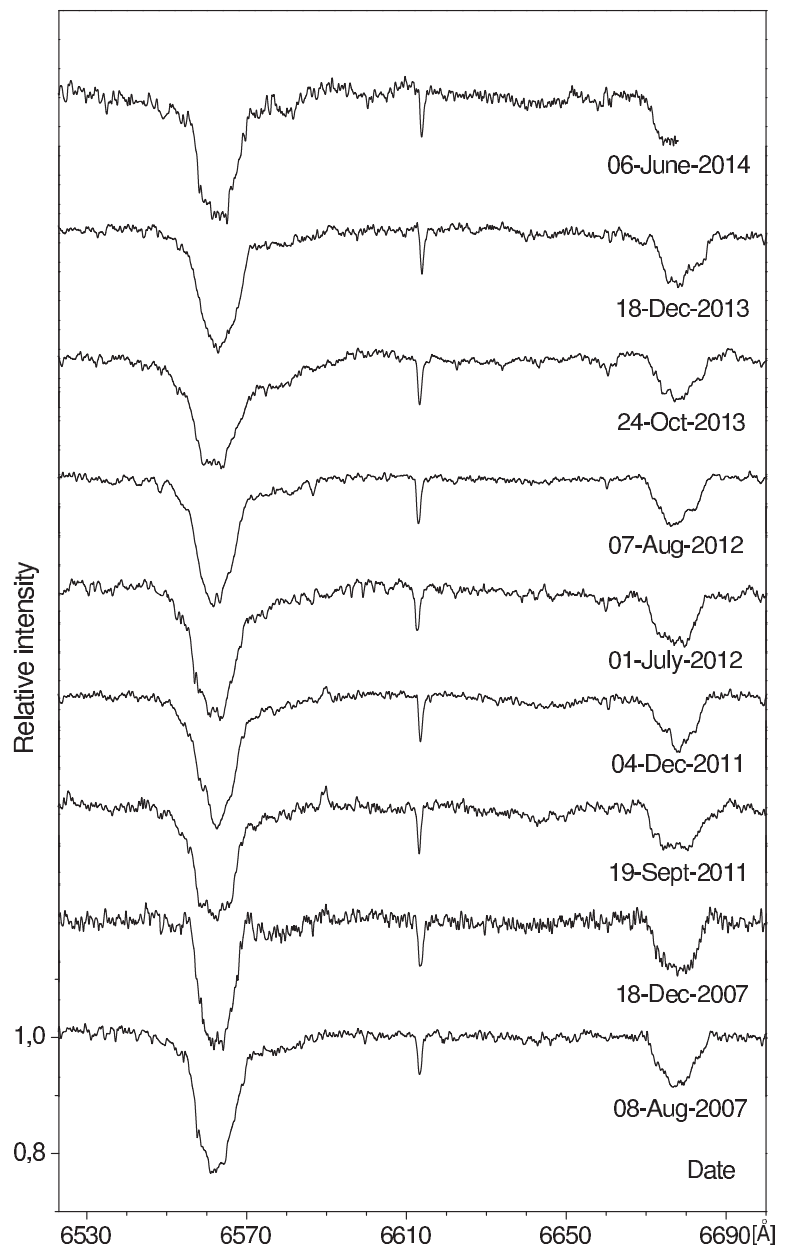

Fig. 2.- A Sample of EM Cep spectra in the B state. our spectra: (relative) intensities of the two emission peaks in the Be state, $\mathrm{I}_{V}$ and $\mathrm{I}_{R}$; intensity of the line centre absorption core, $\mathrm{I}_{a b s}$; equivalent width (EW) and FWHM of the emission and absorption features; and the wavelengths of the emission peaks in the Be state, $\lambda_{V}$ and $\lambda_{R}$. All of these parameters for each observation date are given in Table 2 . In the B state, only the absorption component is present, and the listed EW is the area measured below the continuum; the FWHM is full width of the absorption line measured at half depth. In the Be states, an emission EW is measured as the area in the wings above the continuum; the FWHM of the emission component is the full width measured at half height of the emission wings, whereas the FWHM of the absorption component refers to the full width at half depth of the absorption core.

These line parameters allowed us to separate three levels of the Be state: (1) a high state with peak intensities above $1.1 ;(2)$ a middle state with peak intensities between 1.05 and 1.1 ; and (3) a low state with peak intensity below 1.05. In fact, the $\mathrm{H} \alpha$ profiles at some low Be states have only barely visible emission "horns" (or horn) above the continuum or on the line wings (see 18 Sept. 2010 in Figure 1). All remaining observations with no visible emission peaks were classified as the B state. The variation of the classified state with time is shown in Figure 3, and EM Cep is seen to spend more time in the B state than in the Be state.

We observed two high Be-state episodes: May 2010 and November-December 2014. The spectra from May 4 and 7, 2010, revealed that the $\mathrm{H} \alpha$ emission increased considerably in the framework of 3 days (see Figure 1 and Table 2).

Our observations from April through August 2013, imply that the Be state lasted at least 4 months, while observations from the second half of 2014 allowed us to follow the transition from the B state to the Be state and its end within 7 months (see Figure 1).

\subsection{The $\mathrm{H} \alpha$ line in the Be state}

The Be state $\mathrm{H} \alpha$ profiles of EM Cep consist of a deep, central absorption core and two emission peaks (red and blue-shifted peaks, R and V) that are almost symmetric with respect to the absorption core (see Figure 1). This structure is sunk in a shallow and very broad (nearly $40 \AA$ ) absorption trough. The absorption core and emission peaks are not precisely in the middle of this broad absorption trough but closer to its short-wavelength part. The bottoms of the $\mathrm{H} \alpha$ absorption cores are asymmetric as their right half is filled-in by emission (Figure 1).

The mean separation between the $\mathrm{R}$ and $\mathrm{V}$ emission 
Table 2: Measured line parameters for $\mathrm{H} \alpha$.

\begin{tabular}{|c|c|c|c|c|c|c|c|c|c|c|}
\hline Date & $\mathrm{I}_{V}$ & $\overline{\mathrm{I}_{R}}$ & $\mathrm{I}_{a b s}$ & $\begin{array}{c}\mathrm{EW}_{e m} \\
(\AA)\end{array}$ & $\begin{array}{c}\mathrm{FWHM}_{e m} \\
(\AA)\end{array}$ & $\begin{array}{c}\lambda_{V}-6500 \\
(\AA)\end{array}$ & $\begin{array}{c}\lambda_{R}-6500 \\
(\AA)\end{array}$ & $\begin{array}{c}\mathrm{EW}_{a b s} \\
(\AA)\end{array}$ & $\begin{array}{c}\mathrm{FWHM}_{a b s} \\
(\AA)\end{array}$ & State \\
\hline 2004 Aug 24 & 1.065 & 1.047 & 0.755 & 0.304 & 14 & 56.49 & 68.11 & 1.153 & 5.8 & Be middle \\
\hline 2004 Aug 25 & 1.05 & 1.044 & 0.764 & 0.148 & 13 & 56.47 & 67.75 & 1.211 & 5.7 & Be middle \\
\hline 2005 Apr 15 & - & - & 0.811 & - & - & - & - & 2.279 & 12.1 & B \\
\hline 2005 July 28 & - & - & 0.809 & - & - & - & - & 2.075 & 11 & B \\
\hline 2005 July 29 & - & - & 0.778 & - & - & - & - & 2.213 & 9.7 & B \\
\hline 2005 Aug 29 & - & - & 0.788 & - & - & - & - & 2.455 & 10.1 & B \\
\hline 2005 Aug 30 & - & - & 0.784 & - & - & - & - & 2.015 & 9.8 & B \\
\hline 2006 Feb 07 & - & - & 0.827 & - & - & - & - & 2.148 & 12.6 & B \\
\hline 2006 June 21 & - & - & 0.808 & - & - & - & - & 1.99 & 11.1 & B \\
\hline 2006 June 22 & - & - & 0.83 & - & - & - & - & 2.11 & 11.2 & B \\
\hline 2006 July 18 & - & - & 0.795 & - & - & - & - & 2.082 & 10.1 & B \\
\hline 2006 July 19 & - & - & 0.814 & - & - & - & - & 2.019 & 10.2 & B \\
\hline 2007 Aug 8 & - & - & 0.764 & - & - & - & - & 2.477 & 10.5 & B \\
\hline 2007 Dec 18 & - & - & 0.792 & - & - & - & - & 1.925 & 10 & B \\
\hline 2007 Dec 19 & - & 1.014 & 0.792 & 0.065 & - & - & 70.435 & 1.659 & 9.1 & Be low \\
\hline 2010 May 04 & 1.16 & 1.169 & 0.852 & 1.248 & 14 & 57.64 & 67.199 & 0.449 & 3.8 & Be high \\
\hline 2010 May 07 & 1.2 & 1.206 & 0.82 & 1.691 & 15.2 & 57.638 & 67.539 & 0.542 & 4.3 & Be high \\
\hline 2010 Sept 18 & 0.961 & 0.999 & 0.796 & 0.116 & 10.2 & 57.868 & 66.238 & 0.531 & 4.5 & Be low \\
\hline 2011 Apr 11 & - & - & 0.82 & - & - & - & - & 2.374 & 12.5 & B \\
\hline 2011 Sept 19 & - & - & 0.812 & - & - & - & - & 2.33 & 11.2 & B \\
\hline 2011 Dec 04 & - & - & 0.775 & - & - & - & - & 2.452 & 10.4 & B \\
\hline 2012 Jan 03 & - & 1.004 & 0.793 & 0.036 & - & - & 70.884 & 2.143 & 11.1 & Be low \\
\hline 2012 Jan 04 & - & 0.983 & 0.777 & 0.02 & - & - & 71.622 & 2.214 & 10 & Be low \\
\hline 2012 Jan 05 & - & 1.013 & 0.783 & 0.033 & - & - & 71.462 & 2.14 & 10 & Be low \\
\hline 2012 June 30 & - & - & 0.795 & - & - & - & - & 2.294 & 10.9 & B \\
\hline 2012 Aug 07 & - & - & 0.781 & - & - & - & - & 2.351 & 10.6 & B \\
\hline 2012 Sept 07 & - & - & 0.796 & - & - & - & - & 2.432 & 11.5 & B \\
\hline 2013 Apr 21 & 1.005 & 1.023 & 0.734 & 0.147 & 16 & 55.606 & 70.48 & 1.667 & 7.2 & Be low \\
\hline 2013 Aug 25 & 1.083 & 1.079 & 0.773 & 0.388 & 12.5 & 55.788 & 65.606 & 0.879 & 4.6 & Be middle \\
\hline 2013 Oct 24 & - & - & 0.82 & - & - & - & - & 2.615 & 11.5 & B \\
\hline 2013 Dec 18 & - & - & 0.803 & - & - & - & - & 2.093 & 11.4 & B \\
\hline 2014 June 06 & - & - & 0.793 & - & - & - & - & 2.169 & 11 & B \\
\hline 2014 July 8 & 1.024 & 1.028 & 0.741 & 0.107 & 15.6 & 55.052 & 68.516 & 1.67 & 6.7 & Be low \\
\hline 2014 July 9 & 1.027 & 1.017 & 0.764 & 0.035 & 14.5 & 55.862 & 68.308 & 1.566 & 7 & Be low \\
\hline 2014 Aug 08 & 1.064 & 1.081 & 0.825 & 0.4 & 13.6 & 56.436 & 67.46 & 0.766 & 5.1 & Be middle \\
\hline 2014 Sept 30 & 1.118 & 1.115 & 0.828 & 0.641 & 15.1 & 57.153 & 67.093 & 0.602 & 4.5 & Be high \\
\hline 2014 Nov 11 & 1.163 & 1.182 & 0.795 & 1.213 & 14 & 57.638 & 67.074 & 0.631 & 3.75 & Be high \\
\hline 2014 Dec 13 & 1.133 & 1.127 & 0.773 & 0.926 & 13 & 58.293 & 67.093 & 0.741 & 4 & Be high \\
\hline 2015 Jan 01 & 1.082 & 1.108 & 0.791 & 0.505 & 12.5 & 57.257 & 66.107 & 0.711 & 4.15 & Be middle \\
\hline
\end{tabular}


peaks is $10 \AA$ at high Be state, $11 \AA$ at middle Be state, and $12-15 \AA$ at low Be state. The different separation of the two emission peaks of $\mathrm{H} \alpha$ (Figure 1) means different velocities in the areas of the strongest $\mathrm{H} \alpha$ emission, i.e. the radii (and Keplerian velocities) of the densest rings of the disk vary with time.

The depth of the absorption core tends to deepen from high to low Be state, while its FWHM changes with the $\mathrm{H} \alpha$ emission strength, being $9-11 \AA$ at low Be state, $4.5-7 \AA$ at middle Be state, and $3-5 \AA$ at high Be state. However, the width of the whole $\mathrm{H} \alpha$ profile (without the very broad, shallow absorption trough) was always the same at $\approx 16 \AA$.

The short-term variability during the $0.8 \mathrm{~d}$ period of the $\mathrm{H} \alpha$ profiles in the $\mathrm{Be}$ state reveals two weak trends: the depth of the absorption core is smaller at phases 0.0 and 0.5 ; and the $\mathrm{V}$ peak is slightly higher than $\mathrm{R}$ peak in phase range $0.81-1.00$ and vice versa in phase range 0.0-0.24. Harmanec (1984) also found the $\mathrm{V} / \mathrm{R}$ ratio of $\mathrm{H} \alpha$ to vary from 0.9 at phase 0.2 to 1.15 at phase 0.4 .

The $\mathrm{H} \alpha$ profiles in the Be states did not reveal any notable trend of long-term, "V/R" variability (see Figure 1 and Table 2): the two emission peaks had almost equal intensities in a third of all Be states. The $\mathrm{V}$ peak was very rarely stronger than the $R$ peak, and the $R$ peak was stronger than the $\mathrm{V}$ peak in about half of the Be states, including low Be states when there was only a small $\mathrm{R}$ peak, probably representing the first/last sign of the beginning/end of the Be state. However, due to the small difference between the intensities of the two emission peaks (up to $3 \%$, Table 2), EM Cep cannot be considered a typical "V/R variable" Be star.

\section{2. $\mathrm{H} \alpha$ in the $\mathrm{B}$ state}

The $\mathrm{H} \alpha$ line of EM Cep line in its B state is wholly in absorption (Figure 2). However the profiles are slightly asymmetric, with a filled-in core and equivalent width of $1.9-2.6 \AA$.

The short-term variability during the $0.8 \mathrm{~d}$ cycle of the $\mathrm{H} \alpha$ profile in the B state is weak (Figure 4) while there is a long-term variability of its depth and symmetry (Figure 2). The spectra of Hilditch et al. (1982) also revealed variability of the symmetry of the absorption Balmer lines $\mathrm{H} \beta$ and $\mathrm{H} \gamma$.

We establish that the total width of the $\mathrm{H} \alpha$ profile in the Be and B states is almost the same (Figure 5). Their difference represents the contribution of the additional $\mathrm{H} \alpha$ emission source at the Be state, a contribution that is obviously different for the low, middle and high Be states. Its shape, illustrated in Figure 5, clearly exhibits a disk-like origin.
The FWHM of the absorption $\mathrm{H} \alpha$ line in B state is up to 3.3 times larger than that of the $\mathrm{H} \alpha$ absorption core at Be state; however, their depths are the same (Table 2 and Figure 5).

\subsection{The He I 6678 line}

The depth of the He I 6678 line was more than two times smaller than that of $\mathrm{H} \alpha$ (see Figures 1 and 2). Its core is also filled-in, but in contrast to $\mathrm{H} \alpha$, the He I 6678 profile is not sunk in a broad trough. Translating its observed width, $\approx \pm 6.25 \AA$ from line centre, into a velocity shift gives $280 \mathrm{~km} / \mathrm{s}$, consistent with the $v \sin i$ estimate of Hilditch et al. (1982) obtained from the lines He I 4388, He I 4471, and Mg II 5581.

The short-term (phase) variability of the He I 6678 profile was more pronounced than that of $\mathrm{H} \alpha$ (see Figure 4): it becomes more symmetric and deeper at phases 0.0 and 0.5. Hilditch et al. (1982) also noted that the He I 4471 line changed more regularly with the phase than the Balmer lines.

We found the following peculiarities in the behaviour of the He I 6678 line in 2005: it was wider and its right branch was red-shifted by around $3 \AA$ in the phase range 0.754-0.874 compared to the other phases, whereas the width and position of $\mathrm{H} \alpha$ remained almost the same; see Figure 6. We noted the same, but weaker, effect in the phase range 0.14-0.23. It is not clear if the whole He I 6678 line is shifted to longer wavelengths or only its right branch.

The strongest $\mathrm{H} \alpha$ emission in May 2010 and November 2014 (high Be states) was accompanied with a weak emission in the He I 6678 line (see Figure 1). Then, its profiles become more symmetric and its absorption core becomes deeper and narrower. These observations represent the first detection of He I emission for EM Cep. Table 3 gives the averaged measured line parameters for He I 6678 at the B and Be states.

\subsection{Comparison of $\mathbf{H} \alpha$ among different types of stars}

In Figure 7, we compare the $\mathrm{H} \alpha$ profile of EM Cep in its Be state (Figure 7) with those of two other disklike stars, FK Com (Kjurkchieva \& Marchev 2005) and the nova-like, cataclysmic star UX UMa (Kjurkchieva et al. 2006), observed with the same equipment. We find that all three $\mathrm{H} \alpha$ profiles have very similar widths and emission peak separations. Moreover, we found that these profiles are similar also to the $\mathrm{H} \beta$ and $\mathrm{H} \gamma$ lines of the cataclysmic SU UMa-type star HT Cas (Catalan 1995), as well as to the $\mathrm{H} \alpha$ lines of some $\mathrm{T}$ Tau-type stars (Muzerolle et al. 1998).

Therefore, we are faced the question as to why the 
Table 3: Averaged line parameters for He I 6678 in the B and Be states.

\begin{tabular}{lcccccc}
\hline \hline State & $\mathrm{I}_{e m}$ & $\mathrm{I}_{a b s}$ & $\begin{array}{c}\mathrm{EW}_{e m} \\
(\AA)\end{array}$ & $\begin{array}{c}\mathrm{EW}_{a b s} \\
(\AA)\end{array}$ & $\begin{array}{c}\mathrm{FWHM}_{e m} \\
(\AA)\end{array}$ & $\begin{array}{c}\mathrm{FWHM}_{a b s} \\
(\AA)\end{array}$ \\
\hline $\mathrm{B}$ & - & 0.914 & - & 0.827 & - & 11.7 \\
$\mathrm{Be}$ & 1.036 & 0.882 & 0.19 & 0.676 & 16.2 & 7 \\
\hline
\end{tabular}

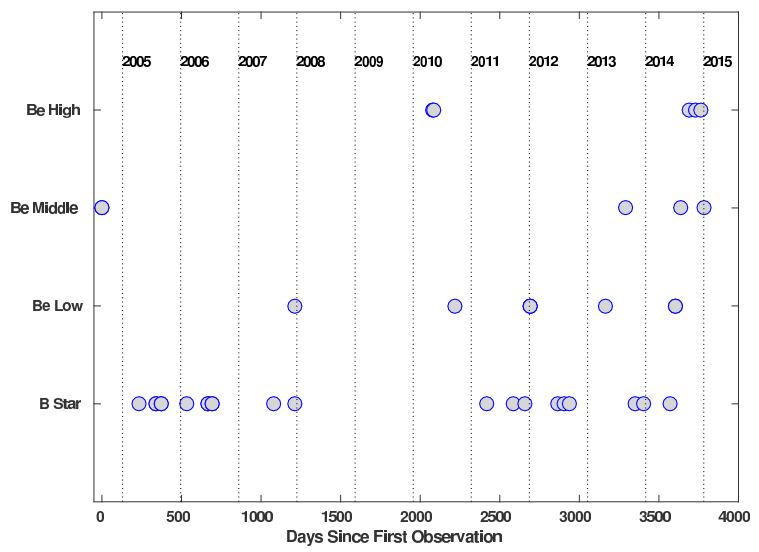

Fig. 3.- State classification of EM Cep (from Table 2) versus time. The year of each observing season is labelled at the top.

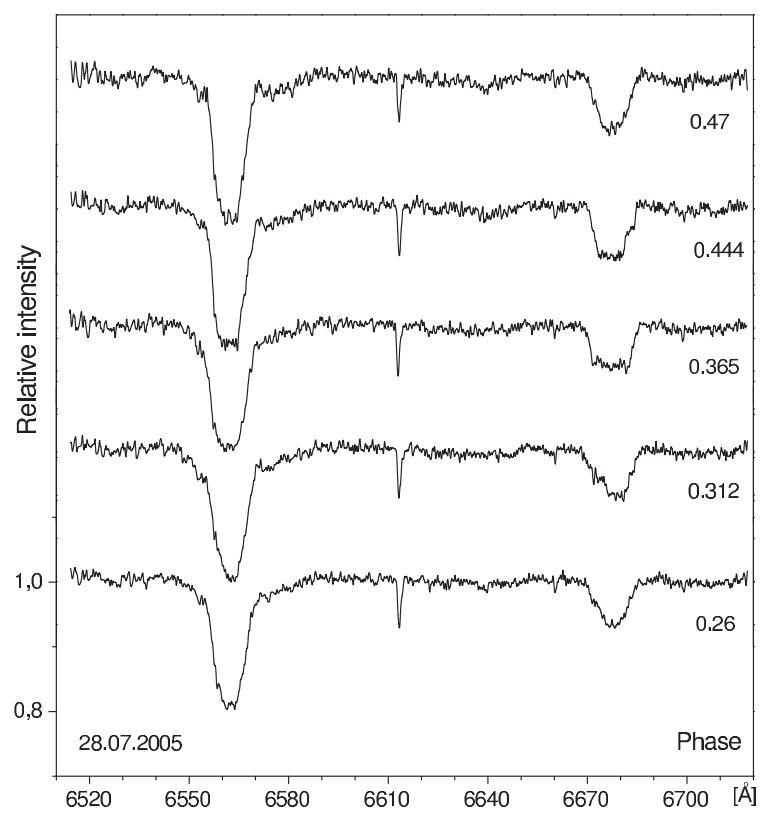

Fig. 4.- Short-term variability of the spectra in the B state on 28 July 2005. The phases from Equation 1 are as indicated.
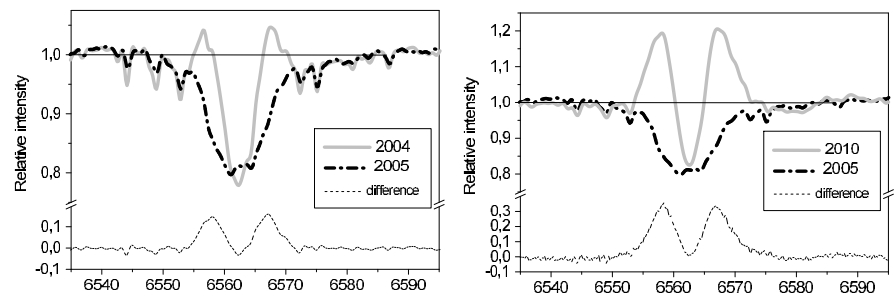

Fig. 5.- $\mathrm{H} \alpha$ in the $\mathrm{B}$ (dash-dot line) and Be states (solid line). The difference between the Be and B state profiles are shown below in each panel and represent the "pure" emission at middle (left panel) and high (right panel) Be states.

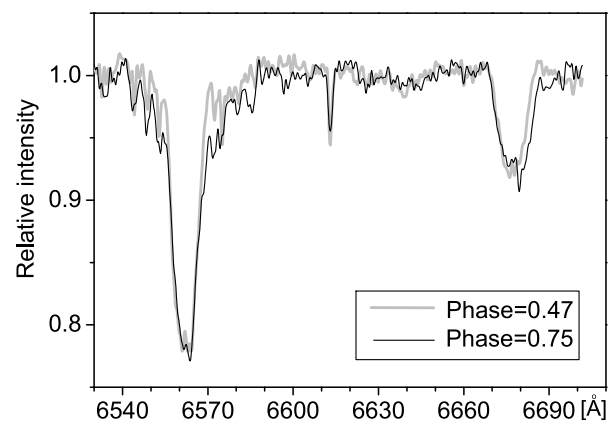

Fig. 6.- The coincidence of the $\mathrm{H}_{\alpha}$ profiles and the difference of the position and the width of the He I lines at phases 0.757 and 0.47 . 
width and emission peak separation of the $\mathrm{H} \alpha$ lines of different types of stars are almost the same. Does this mean that there is some mechanism of creating of similar disk structures with close velocities in the different types of stars?

It should be noted that the Herbig Ae/Be stars are also intermediate mass stars with circumstellar disks (Reipurth et al. 1996; Cauley \& Johns-Krull 2015). However, most of them exhibit considerably stronger $\mathrm{H} \alpha$ emission than EM Cep and their profiles are strongly asymmetric, with considerable differences between the $\mathrm{V}$ and $\mathrm{R}$ intensities). We have found only two Herbig Ae/Be stars with $\mathrm{H} \alpha$ profiles similar to those of EM Cep: AK Sco (see Figure 1 in Reipurth et al. 1996) and CQ Tau (see Figure 19 in Cauley \& Johns-Krull 2015).

\section{Modelling of the $\mathrm{H} \alpha$ profiles of EM Cep}

The 2004-2015 H $\alpha$ spectra of EM Cep seem very similar to typical Be stars in terms of strength and morphology of the emission profile and in the longterm time variation of its strength (for examples of Be star spectra, see Dachs 1988). The $\mathrm{H} \alpha$ profile of EM Cep in its Be state shows approximate symmetry between the red and blue emission in the wings over the duration of the observations. In the maximum $\mathrm{Be}$ state observed for EM Cep (7 May 2010), the H $\alpha$ shell parameter, defined as the average flux in the emission peaks divided by the flux at line centre, is $\sim 1.5$, just satisfying the definition of a Be shell star (Hanuschik 1996). Hence, the target is likely viewed at a high inclination $\left(i>70^{\circ}\right)$ relative to the common rotation axis of the star and circumstellar disk so that the observer's line of sight to the star first passes through the disk.

For this section, we will assume that EM Cep is rapidly rotating, main sequence, $\mathrm{B}$ star surrounded by a thin circumstellar disk in Keplerian rotation (Porter $\&$ Rivinius 2003). Such a model has been widely and very successfully used to model the emission lines, infrared excesses, polarization, and interferometric images of many Be stars (Rivinius et al. 2013a).

We modelled the $\mathrm{H} \alpha$ line of EM Cep as originating from such a circumstellar disk surrounding a B1V star whose parameters are given in Table 2 . The equatorial surface density of the disk was assumed to decrease as a power-law with the radial distance from the star's rotation axis, $R$, as

$$
\sigma(R)=\sigma_{0}\left(\frac{R_{\mathrm{eq}}}{R}\right)^{m}
$$

where $R_{\text {eq }}$ is the star's equatorial radius. We have considered 12 base disk surface densities, $\sigma_{0}=0.001$,
$0.003,0.006,0.010,0.033,0.066,0.100,0.333,0.666$, $1.000,3.333,6.666$, and $10.000 \mathrm{~g} \mathrm{~cm}^{-2}$, and five powerlaw indexes, $m=1.0,1.5,2.0,2.5$, and 3.0. Thus, a total of 60 disk density models were considered.

We have used the code BEDISK (Sigut \& Jones 2007) to compute the temperature structure of the disk for each of the 60 density models. BEDISK assumes that the sole energy input into the disk is the central star's photoionizing radiation field, and it enforces radiative equilibrium by balancing the processes of heating and cooling in a gas of solar chemical composition consisting of the 9 most abundance elements (and their ions). BEDISK produces both the temperature distribution in the disk and atomic level populations that can be used later to formally solve the radiative transfer equation to predict spectral lines of interest, such as $\mathrm{H} \alpha$. BEDISK assumes an axisymmetric disk and returns the disk temperature $T(R, Z)$, where $Z$ is the height above or below the equatorial plane.

To transform the surface density (Eq. (2)) into the corresponding mass density $\rho(R, Z)$, it was assumed that at each radial distance $R$, the disk is in vertical hydrostatic equilibrium with a scale height $H$ parametrized by a single temperature $T_{0}$ as

$$
H=\left(\frac{2 R_{*}^{3} k T_{0}}{G M_{*} \mu_{m} m_{\mathrm{H}}}\right)^{1 / 2}\left(\frac{R}{R_{*}}\right)^{3 / 2} \equiv \beta\left(T_{0}\right) R^{3 / 2} .
$$

Here $M_{*}$ and $R_{*}$ are the mass and radius of the central star, and $\mu_{m}$ is the mean-molecular weight of the gas in the disk. The hydrostatic temperature for EM Cep's disk was set to $T_{0}=0.6 T_{\text {eff }}$ (Sigut et al. 2009), or $15600 \mathrm{~K}$. This choice gives $H / R_{*}=0.037$ at the inner edge of the disk. With this scale height, the mass density in the disk is given by

$$
\rho(R, Z)=\rho_{o}\left(\frac{R_{*}}{R}\right)^{n} e^{-\left(\frac{Z}{H}\right)^{2}},
$$

Table 4: Adopted stellar parameters for EM Cep.

\begin{tabular}{ll}
\hline \hline Parameter & Value \\
\hline $\operatorname{Mass}^{\mathrm{a}}\left(M_{\odot}\right)$ & 12.5 \\
Polar Radius $^{\mathrm{a}}\left(R_{\odot}\right)$ & 6.3 \\
Equatorial Radius $^{\mathrm{a}}\left(R_{\odot}\right)$ & 9.45 \\
${\text { Luminosity }\left(L_{\odot}\right)}$ & $1.6 \times 10^{4}$ \\
$v_{\text {frac }}$ & 0.95 \\
$\mathrm{~T}_{\text {eff }}(\mathrm{K})^{b}$ & 26,000 \\
$\log (g)^{b}$ & 4.0 \\
\hline
\end{tabular}

Notes.

a Adopted from Townsend et al. (2004).

b As defined by the luminosity and polar radius. 


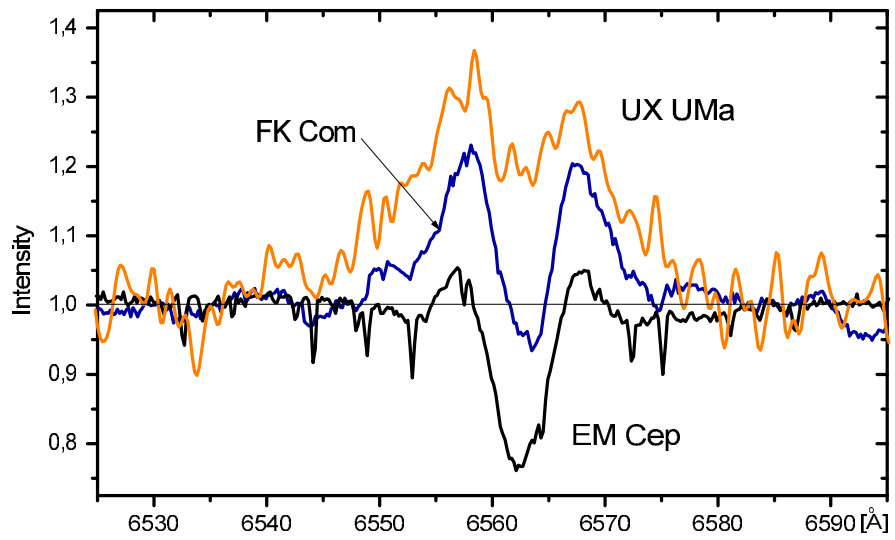

Fig. 7.- The similarity of the $\mathrm{H} \alpha$ lines of UX UMa, FK Com and EM Cep at Be state, observed with the same equipment

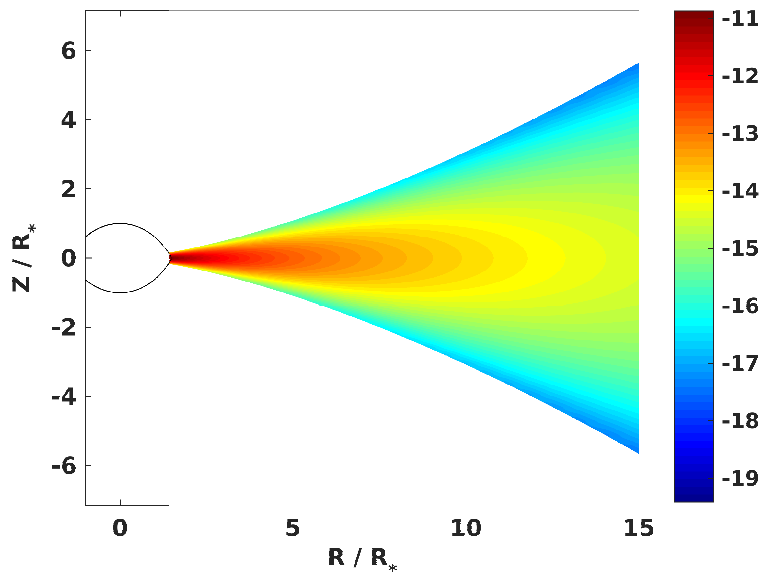

Fig. 8. - The BEDISK disk density model corresponding $\sigma_{0}=0.666 \mathrm{~g} \mathrm{~cm}^{-2}$ and $m=2.0$. The colours represent $\log \rho(R, Z)$ according to the colour bar on the right, where the density is in units of $\mathrm{g} \mathrm{cm}^{-3}$. The outline of the central, distorted star is shown on the left. with

$$
\rho_{0}=\frac{\sigma_{0}}{\sqrt{\pi} \beta\left(T_{0}\right) R_{*}^{3 / 2}},
$$

and

$$
n=m+\frac{3}{2}
$$

As rapid rotation of the central star is though to be a key driver of the Be phenomena (Rivinius 2013b), a gravitationally darkened and a distorted central star should be considered (McGill et al. 2011). Ejection of the circumstellar disk may be linked to episodes of near critical rotation that occur naturally in hydrodynamic models of rapidly rotating B type stars (Granada et al. 2013). For EM Cep, we assumed an equatorial rotation velocity of $95 \%$ of the critical velocity. The distortion of the stellar surface due to the rapid rotation was computed in the Roche approximation (Collins 1966) and the temperature variation from the pole to equator (i.e. the gravitational "darkening") was computed with the Espinosa Lara \& Rieutord (2011) formalism.

The assumption of near critical rotation for EM Cep, coupled with the likely large viewing inclination of the disk (noted above and found from the detailed modelling below), seems to lead to an inconsistency with EM Cep's $v \sin i$ of $\approx 280 \mathrm{~km} \mathrm{~s}^{-1}$. However, strong gravitational darkening is known to lead to a systematic underestimation of $v \sin i$ based on spectral lines because the most rapidly rotating portions of the stellar disk are darker (see Townsend et al. 2004). Finally, we note that the stellar $v \sin i$ has only a small effect on the shape of the strongly Stark-broadened photospheric $\mathrm{H} \alpha$ line.

The density structure of a disk with parameters $\sigma_{0}=0.666 \mathrm{~g} \mathrm{~cm}^{-2}$ and $m=2$ is shown in Figure 8, and the corresponding disk temperature distribution obtained by BEDISK is shown in Figure 9. The circumstellar disk is far from isothermal, with a cool, inner region close to the star resulting from the large optical depths along all rays back to the central star. The strong temperature variation across the surface of the star, from $T_{\text {eff }}=27200 \mathrm{~K}$ at the pole to $T_{\text {eff }}=18300 \mathrm{~K}$ at the equator, is not shown in Figure 9 in order to highlight the (lower) temperatures in the disk. Discussion of the temperature structure of Be star disks can be found in Sigut \& Jones (2007), McGill et al. (2011, 2012).

To compute predicted $\mathrm{H} \alpha$ line profile corresponding to each of the disk density models, the BERAY code of Sigut (2011) was used. BERAY formally solves the equation of radiative transfer along rays threading the star-disk system directed at the observer. Rays that terminate on the stellar surface use an LTE, photospheric, $\mathrm{H} \alpha$ profile, appropriate to the local $T_{\text {eff }}$ and 


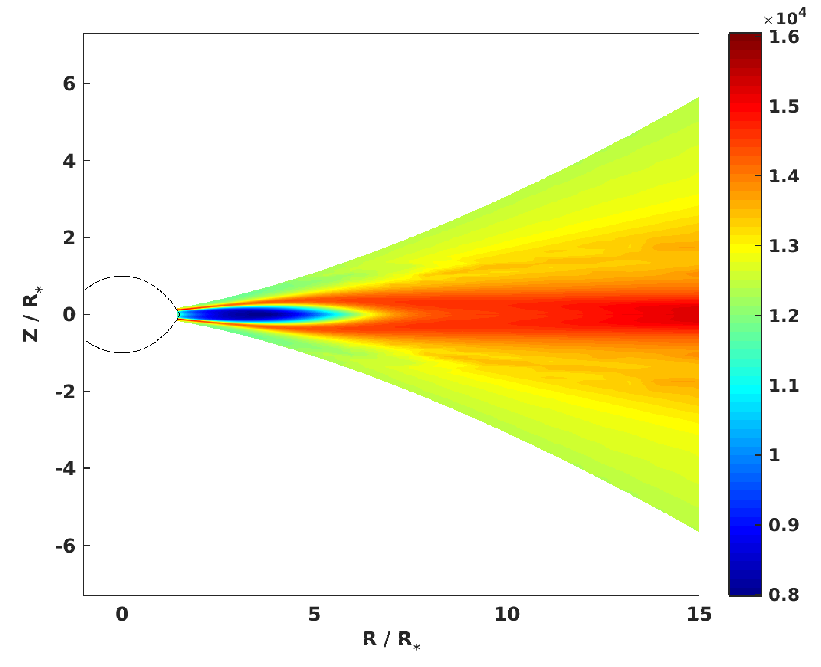

Fig. 9. - The calculated BEDISK temperatures corresponding $\sigma_{0}=0.666 \mathrm{~g} \mathrm{~cm}^{-2}$ and $m=2.0$. The colours represent $T(R, Z)$ in degrees Kelvin according to the colour bar on the right. The outline of the central, distorted star is shown, but not the temperature variation across its surface.
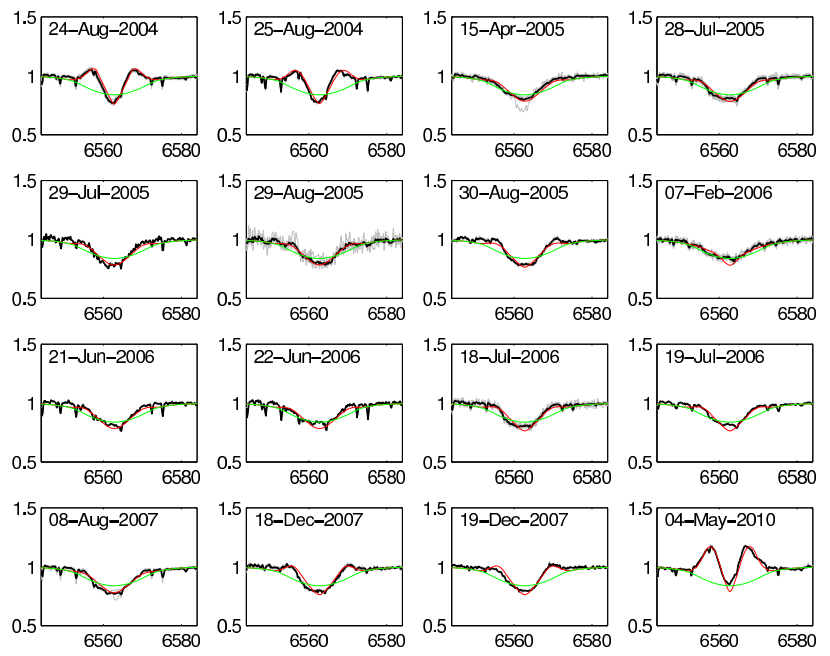

Fig. 10.- The observed $\mathrm{H} \alpha$ profiles (black), the bestfitting BERAY model profiles, and photospheric $\mathrm{H} \alpha$ profiles for the central star (green) for spectra between 24 August 2004 and 4 May 2010.
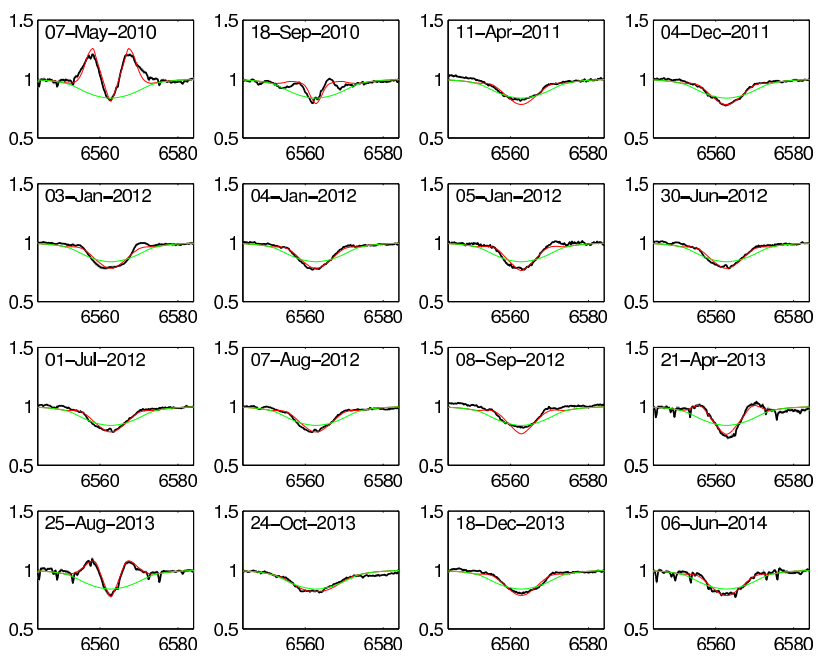

Fig. 11. - Same as Figure 8 but for spectra between 7 May 2010 and 6 June 2014.
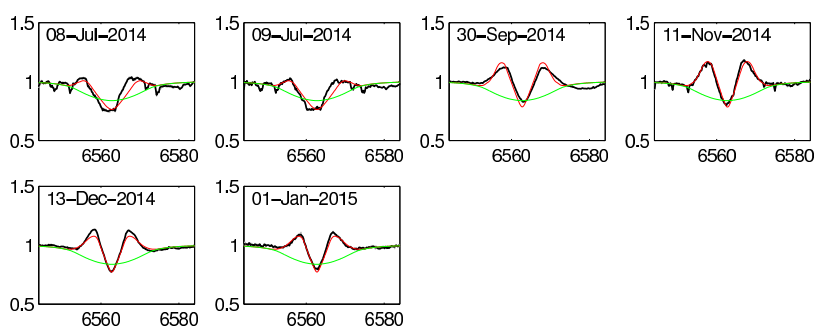

Fig. 12.- Same as Figure 8 but for spectra between 6 July 2014 and 1 January 2015. 
$\log g$ on the stellar surface as the boundary condition, shifted by the radial velocity of that patch. Rays that pass entirely through the disk use a zero boundary condition. The BERAY modelling adds two additional parameters: the viewing inclination of the system (values of $i=18,31,45,60,72,75,78,81,84,87$ and $90^{\circ}$ were used) and the outer disk radius (values of $R_{d}=6$, 12,25 and $50 R_{*}$ were considered). In all cases, the circumstellar disk was assumed to start at the stellar surface, i.e. $R=R_{\mathrm{eq}}$, and extend to $R_{d}$.

In total, a library of $2640 \mathrm{H} \alpha$ profiles was computed for comparison with the observations. All profiles in the library were convolved down to a resolution to match the observations. To fit the appropriate model to each observation, a median observed $\mathrm{H} \alpha$ profile was created for each observing date, and this profile was then compared to the $\mathrm{H} \alpha$ library. The best-fit profile was chosen by minimizing

$$
\mathcal{F} \equiv \frac{1}{N} \sum_{i} \frac{\left|F_{i}^{\mathrm{mod}}-F_{i}^{\mathrm{obs}}\right|}{F_{i}^{\mathrm{obs}}}
$$

where the sum over $i$ is for all $N$ wavelengths in the range $6550 \leq \lambda_{i} \leq 6570$.

In total, 38 such best-fit profiles were generated for the 38 individual days of data, spanning observations from 2004 through 2015. Initially the viewing inclination was left as a free parameter, and the results showed that a value of $i=78^{\circ}$ best fit the emission $\mathrm{H} \alpha$ profiles. All fits were then rerun with the inclination fixed at $78^{\circ}$. Thus the best-fit model for each observed profile gives values for $\sigma_{0}, m$, and $R_{d}$ at that date.

Figures 10 through 12 show the individual best-fits from 24 Aug 2004 through 1 Jan 2015. Overall the quality of the fits is good with the peak separations, central depths, and overall widths of the $\mathrm{H} \alpha$ profile generally reproduced. Close examination of the fits reveal low-level asymmetries and core emission, that cannot be captured by the models because the BEDISK/BERAY modelling assumes an axisymmetric disk and the synthetic $\mathrm{H} \alpha$ profiles are symmetric about line centre. Also shown in each panel of Figures 10 through 12 is the model photospheric $\mathrm{H} \alpha$ profile corresponding to the star alone.

Figure 13 summarizes all of the $\mathrm{H} \alpha$ line profile modelling. Shown is the model fit to the median profile of each identified state of EM Cep (B, Be low, Be middle, and Be high) given in Table 2. The disk parameters corresponding to low, middle and high Be states are given in Table 5. The obtained ranges of values of $\sigma_{0}$, $m$, and $R_{d}$ for the different states of the targets are within those typically found for the Be stars (Silaj et al. 2010, Silaj et al. 2014).
The disk density parameters for each fit to an observed $\mathrm{H} \alpha$ profile can be used to estimate the total mass in the $\mathrm{H} \alpha$ disk as a function of observing date. The outer radius of the disk used for these mass estimates is set to be $\min \left(R_{d}, 20 R_{*}\right)$ as the typical size of the $\mathrm{H} \alpha$ formation region in large disks is $\approx 20$ stellar radii (Grundstrom \& Gies 2006). Because values for the index $m$ in Equation 2 that match the observed profiles are large, $m \geq 2.5$, the resulting disk masses are not particularly sensitive to the exact value of outer disk radius assumed. To get some estimate of the uncertainties involved, disk masses were estimated for the top 5 fitting $\mathrm{H} \alpha$ line profiles for each observed line (i.e. the 5 models with the lowest $\mathcal{F}$ ). The symbol at each date is the mean disk mass with a $1 \sigma$ variation shown.

Figure 14 shows the $\mathrm{H} \alpha$ disk mass of EM Cep as a function of time for the entire observational data set. It is seen to vary by about an order of magnitude, between a low state of $\log \left(M_{D} / M_{*}\right)<\approx-11.0$ and $\mathrm{Be}$ state of $\log \left(M_{D} / M_{*}\right) \approx-10.0$. Only the exclusive low disk mass of $\log \left(M_{d} / M_{*}\right)=-11.25$ for 24 Oct 2013 deviates considerably from the rest values.

Figure 14 can also be used to estimate the massloss rate for EM Cep. Between the beginning of June 2014 and the end of September, 2014, the mass of the disk increases by about $\approx 10^{-9} \mathrm{M}_{\odot}$ giving a massloss rate of approximately $\approx 3 \times 10^{-9} \mathrm{M}_{\odot} \mathrm{yr}^{-1}$. This value is roughly consistent with the range of values found observationally. Based on the IR excess of Be stars, Waters et al. (1987) estimated mass-loss rates (based on a simple, pole-on disk model) of between $\approx 7 \times 10^{-9}$ and $\approx 2 \times 10^{-8} \mathrm{M}_{\odot} \mathrm{yr}^{-1}$. Based on hydrodynamical modelling of the optical light curve of the Be star $28 \mathrm{CMa}$, Carciofi et al. (2012) estimate a mass-loss rate of $3.8 \times 10^{-8} \mathrm{M}_{\odot} \mathrm{yr}^{-1}$. Theoretically, Granada et al. (2013) suggest mass-loss rates in the range of $10^{-11}$ to $10^{-7} \mathrm{M}_{\odot} \mathrm{yr}^{-1}$ for stars losing mass during episodes of critical rotation.

\section{Conclusions}

The main results of our study of EM Cep in its B and Be state may be summarized as follows:

Table 5: Disk parameters for the Be states of EM Cep.

\begin{tabular}{lllll}
\hline \hline State & $\begin{array}{l}\sigma_{0} \\
\left(\mathrm{~g} \mathrm{~cm}^{-2}\right)\end{array}$ & $m$ & $R_{d} / R_{\mathrm{eq}}$ & $M_{d} / M_{*}$ \\
\hline Low Be & 0.333 & 3.0 & 12.0 & $3 \times 10^{-11}$ \\
Middle Be & 0.333 & 2.5 & 25.0 & $5 \times 10^{-11}$ \\
High Be & 0.666 & 2.5 & 25.0 & $1 \times 10^{-10}$ \\
\hline
\end{tabular}



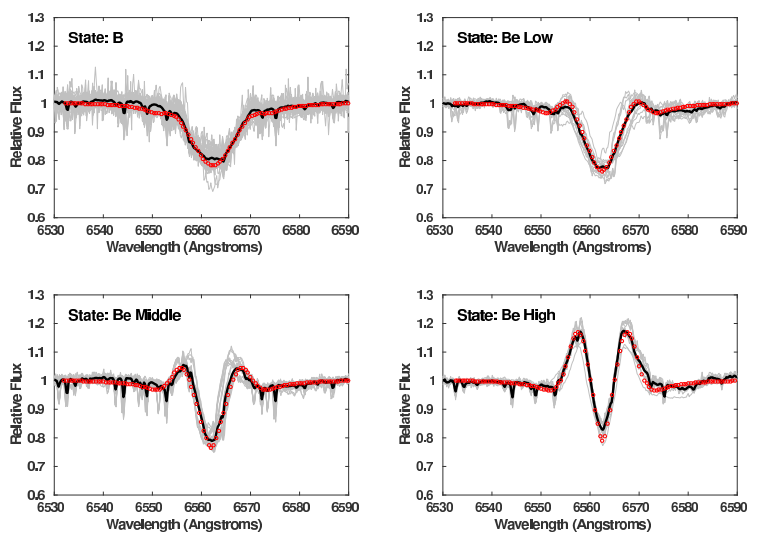

Fig. 13.- Summary of $\mathrm{H} \alpha$ fits according to disk state. In each panel, the $\mathrm{H} \alpha$ line profiles corresponding to the given state are shown as light grey lines; the dark solid line is the median profile, and the red circles are the best-fit model profile with disk parameters given in Table 3. .

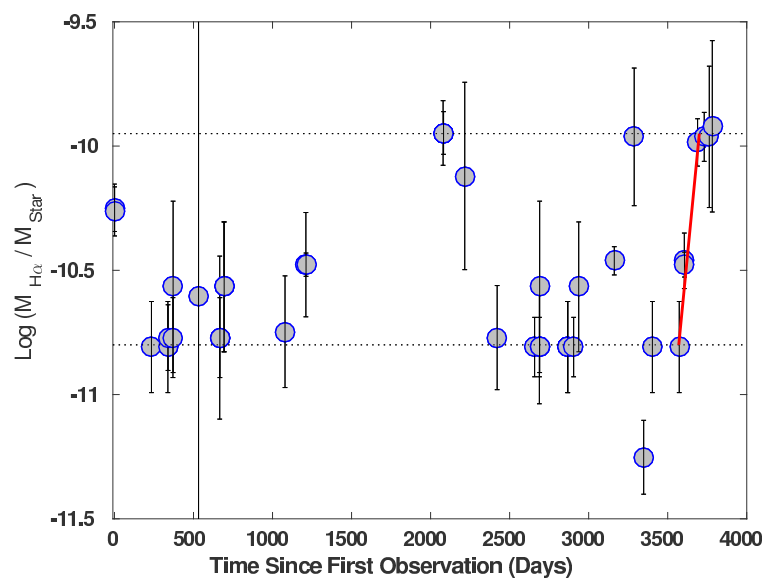

Fig. 14.- Mass of the $\mathrm{H} \alpha$ disk of EM Cep as a function of time from the best-fit density models. Shown for each date are the mean and $1 \sigma$ variation of the masses from the top 5 fitting $\mathrm{H} \alpha$ profiles. The horizontal dotted lines give the approximate values for the high and low mass disk states. The solid red line connects the 3 dates used to estimate the mass loss rate during this period (see text).
1. The different levels of $\mathrm{H} \alpha$ emission were formally separated as initial, middle and high Be states. We detected that the $\mathrm{H} \alpha$ emission increased considerably in the framework of several days at the highest Be state.

2. Eleven years of spectral observations, 2004-2015, reveal that the target stay in the B state exceeds that in the Be state and that the transition from $\mathrm{B}$ to Be to B state again lasted up to 7 months.

3. The spectra of EM Cep within a single night do not change considerably.

4. The $\mathrm{H} \alpha$ profile of EM Cep in the Be state is doubly-peaked with a central absorption core and two emission wings. The ratio of the emission peak flux and the central core flux in the Be state nominally identify EM Cep as a Be shell star. The separation between the emission peaks varied in the range $12-15 \AA$ during the seasons. The $\mathrm{H} \alpha$ profiles reveal weak long-term "V/R" variability.

5. The spectral lines in our spectral range do not reveal any apparent Doppler shifts in both the B and Be state.

6. The FWHM of the absorption $\mathrm{H} \alpha$ line in the $B$ state is several times larger than that of the absorption core of the $\mathrm{H} \alpha$ line at Be state, while their depths are the same. The total width of the whole $\mathrm{H} \alpha$ profile at $\mathrm{Be}$ and $\mathrm{B}$ states are almost the same.

7. The profiles of the absorption line He I 6678 exhibit more remarkable short-time variability than $\mathrm{H} \alpha$. We report the first detection of weak emission in the He I 6678 line during the highest Be state in May 2010.

8. We established the widening and red-shift of the He I 6678 line in the phase range 0.754-0.874 during which the position of the $\mathrm{H} \alpha$ line remained constant.

9. We found similarity in widths and emission peak separations of the $\mathrm{H}_{\alpha}$ line of EM Cep at Be state and those of the disk-like star FK Com and cataclysmic nova-like star UX UMa. This may imply existence of gaseous disks with similar parameters for different types of objects.

Finally, the classical Be star model can satisfactorily reproduce the $\mathrm{H} \alpha$ line profiles of EM Cep with disk density parameters and disk masses that are typical of the Be stars. In its high Be state, the mass of EM Cep's 
$\mathrm{H} \alpha$ disk is $\approx 10^{-10} M_{*}$ and a disk ejection episode observed in 2014 was estimated to imply a mass-loss rate of $\approx 3 \times 10^{-9} \mathrm{M}_{\odot} \mathrm{yr}^{-1}$.

\section{Acknowledgements}

The research was supported partly by funds of project RD-08-81 of Shumen University. TAAS wishes to acknowledge support from the Canadian Natural Sciences and Engineering Research Council (NSERC).

Facilities: National Astronomical Observatory at Rozhen, Bulgaria 


\section{REFERENCES}

Bakos, Gustav A.; Tremko, Jozef, 1975, JRASC, 69, 307

Balona L., 1990, MNRAS 245, 92

Bjorkman, J. E.; Carciofi, A. C., 2005, ASPC, 337, 75

Briot D., 1986, A \& A, 163, 67

Carciofi, A. C.; Bjorkman, J. E., 2006, ApJ, 639, 1081

Carciofi, A. C., Okazaki, A. T., Le Bouquin, J.B., et al. 2009, A \& A, 504, 915

Carciofi, Alex C., 2011, in IAU Symp. 272, Active OB Stars: Structure, Evolution, Mass Loss, and Critical Limits, ed. C. Neiner et al. (Cambridge: Cambridge Univ. Press), 325

Carciofi, Alex C.; Bjorkman, Jon E.; Otero, Sebastin A.; Okazaki, Atsuo T.; Stefl, Stanislav; Rivinius, Thomas; Baade, Dietrich; Haubois, Xavier, 2012, ApJ, 744L, 15

Catalan M., 1995, PhD thesis, The University of Sussex

Cauley P., Johns-Krull C., 2015, ApJ, 810, 5

Collins, G. W., 1966, ApJ, 146, 915

Cranmer, S. R., 2005, ApJ, 634, 585

Dachs, J.; Kiehling, R.; Engels, D., 1988, A \& A, 194, 167

Dougherty, S. M.; Waters, L. B. F. M.; Burki, G.; Cote, J.; Cramer, N.; van Kerkwijk, M. H.; Taylor, A. R., 1994, A \& A, 290, 609

Espinosa Lara, F., Rieutord, M., 2011, A \& A, 533, A43

Gehrz, R. D.; Hackwell, J. A.; Jones, T. W., 1974, ApJ, 191, 675

Granada, A., Ekstrom, S., Georgy, C., Krticka, J., Owocki, S., Meynet, G., Maeder, A. 2013, A \& A, $553, \mathrm{~A} 25$

Grundstrom, E. D., Gies, D.R., 2006, ApJL, 651, L53

Hanuschik, R. W., 1996, A \& A, 308, 170

Harmanec P., 1984, BAICz 35, 193

Hubert, A. M.; Floquet, M., 1998, A \& A 335, 565

Hilditch R., McLean B., Reid I., 1982, MNRAS 200, 1153
Johnston K., 1970, PASP 82, 1093

Kaiser, D., 1989, A \& A, 222, 187

Kjurkchieva, D. P.; Marchev, D. V., 2005, A \& A, 434, 221

Kjurkchieva D., Marchev D., Khruzina T., Djurasevic G., 2006, Ap\&SS 306, 217

Kochiashvili, N. T., 1999, Ap, 42, 399

Kochiashvili, N.; Natsvlishvili, R.; Bakis, H.; Tanriver, M., 2007, 2007, A \& AT, 26, 113

Kreiner J., Kim C., Nha II-Seong, 2001, An atlas of OC Diagrams of eclipsing Binary stars, Krakow Pedagogical University Press

Lee, Umin; Osaki, Yoji; Saio, Hideyuki, 1991, MNRAS, 250, 432

Lynds, C. R., 1959, ApJ, 129, 674

Mars M., Aas T., Harvig V., 2010, ASP Conference Series, Vol. 435, 131

Martayan, C.; Frmat, Y.; Hubert, A.-M.; Floquet, M.; Zorec, J.; Neiner, C., 2007, A \& A, 462, 683

McGill, M. A., Sigut, T. A.A., Jones, C. E., 2012, ApJ Supp. Ser., 204, 2

McGill, M.A., Sigut, T.A.A., Jones, C. E., 2011, ApJ, 743,111

McSwain, M. V.; Gies, D. R., 2005, ASPC, 337, 270

Meilland, A.; Stee, P.; Vannier, M.; Millour, F.; Domiciano de Souza, A.; Malbet, F.; Martayan, C.; Paresce, F.; Petrov, R. G.; Richichi, A.; Spang, A., 2007, A \& A, 464, 59

Meilland, A.; Millour, F.; Kanaan, S.; Stee, Ph.; Petrov, R.; Hofmann, K.-H.; Natta, A.; Perraut, K., 2012, A \& A, 538A, 110

Mermilliod, J. C., 1982, A \& A, 109, 48

Merrill P., Humason M., Burwell C., 1932, ApJ 76, 156

Molik P, Wolf M., 2004, Balt.Astr. 13, 145

Muzerolle, James; Hartmann, Lee; Calvet, Nuria, 1998, AJ, 116, 455

Neiner, C.; de Batz, B.; Cochard, F.; Floquet, M.; Mekkas, A.; Desnoux, V., 2011, AJ, 142, 149

Okazaki, Atsuo T., 1991, PASJ, 43, 75 
Okazaki, Atsuo T., 2001, PASJ, 53, 119

Oudmaijer, Ren D.; Parr, Andrew M., 2010, MNRAS, 405, 2439

Plaskett, J. S., \& Pearce, J. A., 1931, Publications of the Dominion Astrophysical Observatory, 5, 1

Porter, John M., 1999, A \& A, 348, 512

Porter, J.M., Rivinius, T., 2003, PASP, 115, 1153

Pribulla T., Rucinski S., 2006, AJ 131, 2986

Pustylnik, I.; Kalv, P.; Aas, T.; Harvig, V.; Mars, M., 2005, ASPC, 335, 321

Rachkovskaia T., 1975, Izv.Kr.Astr.Obs., 53, 168

Rachkovskaia T., 1976, Izv.Kr.Astr.Obs., 55, 100

Rachkovskaia T., 1977, Izv.Kr.Astr.Obs., 56, 11

Raddi, R.; Drew, J. E.; Steeghs, D.; Wright, N. J.; Drake, J. J.; Barentsen, G.; Fabregat, J.; Sale, S. E., 2015, MNRAS, 446, 274

Reipurth B., Pedrosa A., Lago M., 1996, A\&AS, 120, 229

Rivinius, T., Carciofi, A. C., Martayan, C., 2013a, A \& ARv, 21, 69

Rivinius, T., 2013b, Astrophys Space Sci Proc., 31, 253

Sigut, T.A.A, Jones, C.E., 2007, ApJ, 668, 481

Sigut, T.A.A, McGill, M.A., Jones, C.E., 2009, ApJ, 699, 1973

Sigut, T.A.A., 2011, IAU Symp. 272, "Active OB Stars: Structure, Evolution, Mass-Loss, and Critical Limits," Neiner, C., Wade, G., Meynet, G., \& Peters, G. (eds), 426

Silaj, J., Jones, C. E., Sigut, T. A. A., Tycner, C., 2014, ApJ, 795, 82

Silaj, J., Jones, C. E., Tycner, C., Sigut, T. A.A., Smith A., D., 2010, ApJS, 187, 228

Slettebak, A., 1985, ApJS, 59, 769

Struve O., 1931, ApJ, 73, 94

Tody, D., 1993 in X, ASP Conference Series 52, 173T

Townsend, R. H. D., Owocki, S. P., Howarth, I. D., 2004, MNRAS, 350, 189
Uesugi A., Fukuda I., 1982, Revised Catalogue of Stellar Rotational Velocities (Univ. of Kyoto, Kyoto, Japan)

Wheelwright, H. E.; Bjorkman, J. E.; Oudmaijer, R. D.; Carciofi, A. C.; Bjorkman, K. S.; Porter, J. M., 2012, MNRAS, 423L, 11

Zasche, P.; Wolf, M.; Hartkopf, W. I.; Svoboda, P.; Uhlar, R.; Liakos, A.; Gazeas, K., 2009, AJ 138, 664

Zorec, J.; Briot, D., 1991, A \& A, 245, 150

Zorec, J.; Briot, D., 1997, A \& A, 318, 443

This 2-column preprint was prepared with the AAS LATEX $_{\mathrm{E}}$ macros v5.2. 